\title{
Robot's social gaze affects conflict resolution but not conflict
} adaptations.

Francesca Ciardo ${ }^{1}$ and Agnieszka Wykowska ${ }^{1 *}$

6 Word count (Abstract \& main text): 7721

7 References: 71

$8 \quad{ }^{*}$ Corresponding author:

9 Agnieszka Wykowska

10 Istituto Italiano di Tecnologia

11 Via Enrico Melen 83

1216152 Genova, Italy

13 Agnieszka.Wykowska@iit.it 
Robot's social gaze affects conflict resolution

\section{Abstract}

15 Robots are a new category of social agents that, thanks to their embodiment, can be used to train and 16 support cognitive skills such as cognitive control. Several studies showed that cognitive control mechanisms

17 are sensitive to affective states induced by humor, mood, and symbolic feedback such as monetary rewards.

18 In the present study, we investigated whether the social gaze of a humanoid robot can affect cognitive 19 control mechanisms. To this end, in two experiments, we evaluated both the conflict resolution and trial-by20 trial adaptations during an auditory Simon task, as a function of the type of feedback participants received 21 in the previous trial from the iCub robot, namely, mutual or avoiding gaze behaviour. Across three 22 experiments, we compared the effect of mutual, avoiding (Exp1 and Exp2), and neutral (Exp3) gaze 23 feedback between screen-based (Exp1) and physically embodied setups (Exp2 and Exp3). Results showed

24 that iCub's social gaze feedback modulated conflict resolution, but not conflict adaptations. Specifically, the

25 Simon effect was increased following mutual gaze feedback from iCub. Moreover, the modulatory effect

26 was observed for the embodied setup in which the robot could engage or avoid eye contact in real-time

27 (Exp2) but not for the screen-based setting (Exp1). Our findings showed for the first time that social 28 feedback in Human-Robot Interaction, such as social gaze, can be used to modulate cognitive control. The 29 results highlight the advantage of using robots to evaluate and train complex cognitive skills in both healthy 30 and clinical populations.

\section{Keywords:}

32 Conflict resolution, Conflict adaptations, Social Gaze, Human-Robot Interaction 
Robot's social gaze affects conflict resolution

\section{Introduction}

Complex cognitive skills in humans are the result of interaction with the environment, including social interactions. Indeed as stated by Humpreys, "[...] cognitive functions have evolved to their high level because they have been driven by the complexities of social living" (Humphreys, 1976, p. 307). Nowadays, social interactions are not only limited to other humans but also include artificial agents, such as robots. The pivotal role that robots can play in promoting and supporting human cognitive skills is demonstrated by the increased interest in developing socially assistive robotics in the last years (Chevalier et al., 2019; Wykowska, 2020). Indeed, as social agents, robots represent a perfect combination of symbolic (e.g. language and communicative gestures) and artifactual instruments that, according to the socio-cultural approach (e.g., Vygotskij, 1932), are necessary to ensure and support the development of cognitive skills such as communication and behaviour regulation (Ciardo \& Wykowska, 2020).

Among cognitive skills, one of the most crucial is cognitive control, which is the ability to adapt our behaviour to maintain and achieve task goals by reducing the cognitive conflict in task execution, when simultaneous and mutually incompatible goal representations competing for a single response are activated. Several studies showed that cognitive conflict can be modulated by either enhancement of processing of task-relevant information (e.g., Egner \& Hirsh, 2005) or by inhibition of task-irrelevant features (e.g., Braver, 2012; Ridderinkhof, 2002). The former refers to the adjustments of task parameters and priority settings occurring at the level of task strategy (e.g. Braver, 2012; Logan, 1985), resulting in a better conflict resolution within a given trial. Namely, resources are allocated to prevent the negative impact of a cognitively demanding event on task performance (e.g., Marini, Chelazzi, \& Maravita, 2013). This form of control differs from online conflict adaptations in performance triggered by the conflict experienced in the previous trial (e.g., Botvinick, Braver, Barch, Carter, \& Cohen, 2001; Braver, 2012).

The mechanisms allowing humans to deal with cognitive conflict have been studied in the literature using tasks that manipulate conflict, such as the Flanker task (Eriksen \& Eriksen, 1974), the Stroop task (Stroop, 1992), the task-switching paradigms (e.g. Braem et al., 2012) or the Simon task (Simon \& Rudell, 1967; for a review see Proctor \& Vu, 2006). In a Simon task, participants are asked to respond to a feature of a target stimulus usually presented visually on the screen. The feature, colour, for example, determines which button a participant is supposed to press (e.g., left button for red targets and right button for green 
Robot's social gaze affects conflict resolution

61 targets). Most importantly, the target stimuli are presented laterally on the screen, and the responses are 62 also lateral. This means that each target will require a spatially corresponding or non-corresponding 63 response (in the example above, if the red target is presented on the left, the response will be spatially 64 corresponding, but if the red target is presented on the right, the response will not be corresponding). 65 Typically, corresponding target-response mappings elicit faster responses (and/or lower error rates) than 66 non-corresponding mapping (e.g. Simon \& Rudell, 1967), although the spatial configuration is completely 67 irrelevant to the task. The correspondence effect in a Simon task is termed the Simon effect (SE), and it 68 has been explained as resulting from a conflict during response selection (e.g., Rubichi \& Pellicano, 2004) 69 between two alternative response codes, one generated based on task instructions and the other 70 automatically activated through pre-existing associations linking a stimulus to its spatially corresponding 71 response (e.g., De Jong, Liang, \& Lauber. 1994). The automatic association between stimulus and 72 response codes has laid the grounds for the Theory of Event Coding (Hommel et al., 2001) which postulates 73 a common code between perception and action. Most importantly, due to the automatic activation of a 74 stimulus-response code that incorporates spatial information, even though it is task-irrelevant, the response 75 selection process is facilitated in corresponding trials, in which the two activated response codes overlap, 76 leading to faster reaction times (RTs). Conversely, conflict occurs in non-corresponding trials in which the 77 irrelevant and the relevant stimulus dimensions activate different response codes, thus impeding RTs. 78 Interestingly, SE is reduced, null, or even reversed for trials that follow a non-corresponding trial, while it is 79 consistently observed for trials following a corresponding trial (e.g., Ciardo, Ricciardelli, \& lani, 2019;

80 Hommel, Proctor, \& Vu, 2004). These trial-by-trial adaptations have been taken as evidence that the conflict 81 experienced in a given trial is accompanied by changes aimed at preventing the recurrence of the conflict 82 in the next trial (for a review, see Mansouri, Tanaka, \& Buckley, 2009; but see also Hommel et al., 2004 for 83 an alternative account).

84 Although the independence of conflict resolution and conflict adaptation mechanisms is still 85 debated (see Egner, Ely, \& Grinband, 2010; Scherbaum, Fischer, Dshemuchadse, \& Goschke, 2011 for an 86 alternative account), they show different developmental strategies and are differently affected by individual 87 and contextual factors. Indeed, evidence shows that individual differences such as age, cognitive style, or 88 psychiatric disorders (e.g. eating or mood disorders) are reflected in conflict resolution, but not in conflict 
Robot's social gaze affects conflict resolution

89 adaptations (Larson, Clawson, Clayson, \& South, 2012; Iani, Stella, \& Rubichi, 2014; lani, Ciardo,

90 Ricciardelli, \& Nicoletti, 2016; Bartholdy, et al., 2017).

91 Another factor to which cognitive control seems to be sensitive is the affective state ${ }^{1}$ induced by reward,

92 humor, and mood (see van Steenbergen, 2015 for a review). For instance, Kanske and Kotz investigated,

93 in a series of studies, whether and how conflict resolution is modulated by the emotional content of the

94 stimuli. Using different types of conflict tasks, the authors showed that both positive and negative words

95 can speed up conflict resolution (i.e. reduced Flanker and Simon effects for negative and positive targets,

96 respectively, Kanske \& Kotz, 2010; 2011;2012). A similar result was reported by Yamaguchi \& Nishimura

97 (2018), who manipulated monetary reward. Specifically, using a Flanker task, the authors showed that

98 conflict was reduced (i.e. smaller Flanker effect) for contingent-reward trials compared to trials in which the

99 reward was randomly assigned (Exp2, Yamaguchi \& Nishimura, 2018). However, this was not the case for

100 conflict adaptations, as the authors showed that both contingent and non-contingent rewards had little effect

101 on trial-by-trial effects, highlighting the dissociation between conflict resolution and conflict adaptation.

102 Online conflict adaptations have also been shown to be modulated by affective states. Padmala and

103 colleagues (2011) presented both neutral and high-arousing negative images (i.e., mutilated bodies) in-

104 between Stroop task trials. The results showed that following negative pictures the usual trial-by-trial

105 adaptations did not occur. Similarly, van Steenberg and colleagues (2010) investigated the effect of

106 positive and negative mood-induction before performing a conflict-evoking Flanker task. Results showed

107 that trial-by-trial adaptations were affected by the pleasure of the induced mood, with a larger reduction in

108 cognitive conflict (i.e. less interference) for subjects assigned to the negative mood condition (i.e., anxious

109 and sad) compared to those assigned to the positive mood group (i.e., calm and happy). Similar results

110 have been also reported from studies that manipulated reward, showing an enhanced cognitive control

111 (i.e. less interference) following negative feedback (i.e. losses or small gains) than following gain

112 feedback (e.g., Braem et al., 2012; Stürmer et al., 2011; Schuch, Zweerings, \& Koch, 2017; but see also

${ }^{1}$ Please note that when talking about affective states we refer to positive or negative states that could be driven both by motivational factors and/ or emotions. 
Robot's social gaze affects conflict resolution

113 Yamaguchi et al.2020 for a series of studies failing in replicating the effect of affective states on trial-by114 trial effects).

115 It has been proposed that affective states (mood, reward, or emotions) seem to enhance cognitive 116 control in an affect-congruent manner (cf. Cabanac, 1992; van Steenberg, 2014). That is, negative affective 117 states might influence cognitive conflict for the demanding cases, i.e. incongruent (conflict) trials, as those 118 also trigger a negative and aversive state (Botvinick, 2007). A recent systematic review by Dignath and 119 colleagues (2020) underlies how the effect of affective states on conflict adaptations is influenced by the 120 way the affective state is induced during the task. While tonic affective states, like mood, showed consistent 121 results in support to the affect-congruent hypothesis, studies that manipulated affective states in a transient 122 way, such as monetary reward on random trials, showed mixed and contradictory results, mostly due to the 123 heterogeneity of the tasks and manipulations across studies (Dignath et al., 2020).

124 The studies reviewed so far manipulated mainly emotions and monetary rewards, focusing more on 125 the distinction between affective states and motivational factors. However, in everyday life during social 126 interactions, we are exposed to several non-verbal social communication signals, such as facial expression, 127 body language, and social gaze, that can induce affective states or, as feedback, modulate our motivation. 128 For instance, when we are taking an exam, a smiling examiner looking towards us can induce a more 129 relaxed state and, thereby, help in focusing on our task.

The social gaze is defined as the use of gaze direction with communicative intent (e.g., Emery, 2000).

131 Two social gaze behaviours that induce affective states are: avoiding and mutual gaze. Converging 132 evidence suggests that real-time mutual gaze increases arousal and evokes a positive affective state (but 133 see also Jarick \& Kingstone, 2015, for evidence on mutual gaze being perceived as socially uncomfortable 134 and aversive). For instance, mutual gaze has been associated with increased skin conductance, heart rate, 135 suppression of alpha activity, and increased engagement compared to avoiding gaze (e.g., Hietanen et al., 136 2008; Pönkänen, Peltola, \& Hietanen, 2011; Kompatsiari et al., 2021a). Interestingly, such a modulation 137 appears to be null or reversed when the social gaze is presented within screen-based setups (Hietanen et 138 al., 2008; Pönkänen, Peltola, \& Hietanen, 2011).

139 In a recent series of studies, Kompatsiari and colleagues showed that similar effects are elicited also 140 in interaction with a robot (e.g., Komptsiari et al., 2018; 2019; 2021a). Specifically, when the humanoid 
Robot's social gaze affects conflict resolution

141 robot iCub (Metta et al., 2010) established real-time mutual gaze it was judged as more engaging and 142 human-like compared to when it avoided eye contact. Similarly, Schellen et al. reported that mutual or 143 avoiding gaze presented after participants' choice in a decision-making task induced a change of strategy 144 in the subsequent trial. The decision-making task was related to giving honest or deceptive feedback to 145 iCub. The authors found that fewer deception choices occurred after trials in which iCub established mutual 146 gaze as a feedback (Schellen, Bossi, \& Wykowska, 2021). In a recent EEG study, Kompatsiari and 147 colleagues examined oscillatory brain response to mutual and averted gaze established by the iCub robot 148 during an attentional cuing task (Kompatsiari, Bossi, \& Wykowska, 2021). Results showed that mutual and 149 avoiding gaze differently affected the desynchronization of alpha-band activity, suggesting that following 150 mutual gaze, participants were inhibiting task-irrelevant information to a lesser extent, relative to averted 151 gaze. Such a result is in line with the hypothesis that cognitive control is reduced in social situations, as the 152 need of monitoring another agent reduces the attentional resources allocated to the task (e.g.Huguet et al., 153 2014). In line with this latter hypothesis, there is evidence showing that conflict effects, such as the Stroop 154 effect, are reduced in the presence of another human or a humanoid agent (Huguet et al.,1999; Spatola et 155 al ., 2018; see Belletier et al 2019 for a review).

156 To summarize, the social gaze is a powerful (often implicit) feedback that in social interactions can 157 induce affective states. The effectiveness of mutual or averted gaze seems to be stronger for real-time 158 (embodied) interactions compared to screen-based setups (Hietanen et al., 2008; Pönkänen, Peltola, \& 159 Hietanen, 2011), and it has been widely replicated in Human-Robot Interaction (HRI) (Kompatsiari et al., $1602018 ; 2019: 2021 a)$. The fact that robotic agents can exert similar effects on cognitive mechanisms to those 161 induced by other humans is recent but consistent evidence across several tasks. For instance, it has been 162 shown that human and non-anthropomorphic robotic agents similarly affect sense of agency (e.g. Ciardo 163 et al., 2020), motor preparation, and attention allocation both at the behavioural and neural levels (Hinz et 164 al., 2021; Komptsiari et al 2018)

165 The increasing interest in developing socially assistive robotics for training purposes (Ciardo \& 166 Wykowska, 2020; Wykowska, 2020) calls for the need to study whether the social signals of a robot can be 167 used as feedback to modulate cognitive control mechanisms. Therefore, the present study, aimed to i) 168 investigate whether using the social gaze of a robot as feedback modulates cognitive control; ii) understand 
Robot's social gaze affects conflict resolution

169 if the social gaze feedback of a robot modulates cognitive control by conflict resolution mechanism (i.e., 170 adjusting task parameters and priority settings before the occurrence of cognitively demanding events), or 171 by affecting also trial-by-trial adaptations; iii) test the impact of physical embodiment. In three experiments, 172 we evaluated both the SE overall and trial-by-trial adaptations as a function of the type of social gaze 173 feedback that iCub exhibited at the end of the previous trial, namely mutual, avoiding, or neutral gaze.

174 We implemented an auditory Simon task (Simon \& Rudell, 1967) to avoid having too much visual 175 information to process while performing the task and to make sure that visual attention was focused on 176 iCub's face. In Exp1 and Exp2, we compared the effect of the social gaze feedback between screen-based 177 setups and setups involving physically present embodied iCub, respectively. In Exp 3, we ran a follow-up 178 control in which the robot was not providing any feedback to the participants. This was done to estimate 179 the baseline magnitude of the SE elicited by our embodied setup.

$180 \quad$ Based on the reviewed literature (Hietanen et al., 2008; Kompatsiari et al., 2018; 2019), we 181 hypothesized that iCub's mutual and avoiding gaze should influence cognitive control mechanisms by 182 inducing affective states. According to the hypothesis that affective states modulate cognitive control in an 183 affect-congruent way (van Steenberg, 2014), and the existing evidence showing that mutual gaze induces 184 positive affective states (Hietanen et al,, 2008; Pönkänen, Peltola, \& Hietanen, 2011; Kompatsiari et al., 185 2021a), the SE was expected to be reduced following avoiding gaze feedback or increased after mutual 186 gaze feedback. Specifically, if the social gaze interacts with conflict resolution, then its modulatory effect 187 should be evident at the task strategy level, resulting in a smaller SE within a given trial. On the other hand,

188 if the social gaze modulates online conflict adaptations, then different trial-by-trial effects should emerge 189 across the two gaze conditions. Concerning the comparisons between screen-based and embodied setups, 190 we hypothesized that the social gaze (mutual or avoiding) should be more effective when manipulated in 191 physical presence and in real-time compared to when it is depicted on a screen. Thus, the modulating effect 192 of social gaze should be stronger for embodied setup (Exp2) compared to the screen-based setup (Exp1).

\section{Experiment 1}

194 Participants. 
Robot's social gaze affects conflict resolution

195 The sample size was estimated via a priori power analysis using G*Power. The analysis yielded a sufficient 196 number of 15 participants for the within-subject design [ $\mathrm{dz}=0.40, \alpha=0.05$, and 1- $\beta=0.80]$.Twenty-one 197 participants (5 males; mean age: $24.4 \pm 3.5$ years) took part in the study. All participants had normal or 198 corrected-to-normal vision and were not informed about the purpose of the experiment. All participants gave 199 their informed written consent. All experiments were conducted under the ethical standards laid down in

200 the 1964 Declaration of Helsinki and were approved by the Local Ethical Committee (Comitato Etico 201 Regione Liguria). The data of one participant have been excluded due to a technical failure of the program.

202 Therefore, data of twenty participants were further analyzed.

\section{Materials and Methods}

\section{Apparatus and Stimuli.}

205 The experiment was carried out in a dimly lit and noiseless room. The participant was seated facing a 22"

206 LCD monitor driven by a $2.90 \mathrm{GHz}$ processor computer at a viewing distance of $60 \mathrm{~cm}$. Stimuli were 'high' $207(800 \mathrm{~Hz})$ or 'low' $(400 \mathrm{~Hz})$ tones presented through Sennheiser HD 569 headphones $(10-28000 \mathrm{~Hz})$. 208 Responses were executed by pressing the ' $q$ ' or the ' $p$ ' keys on the QWERTY keyboard with the left or the 209 right index finger, respectively. Two videos showed the iCub robot establishing real-time mutual or avoiding 210 gaze (from a participant's perspective). The keyboard was located centrally with respect to the body midline.

211 Stimulus presentation, response timing, and data collection were controlled by Psychopy software

212 (v.2020.1.3)

213 Procedure.

214 Participants were instructed to respond as quickly and as accurately as possible to the tone pitch presented 215 to one of their ears through either left or right headphones. Participants were asked to ignore the tone's 216 spatial location. Half of the participants responded to the high tone by pressing the "q" key and to the low 217 tone by pressing the "p" key, the other half experienced the opposite stimulus-response mapping. Each trial 218 began with the presentation of a white fixation cross in the center of a black screen for $900 \mathrm{~ms}$, then the 219 fixation cross turned to yellow for $1100 \mathrm{~ms}$. $200 \mathrm{~ms}$ after the fixation cross changed colour the tone was 220 played, see D'ascenzo et al. (2018) for a similar procedure. Given that evidence showed that left and right 221 auditory stimuli produce a decreasing Simon effect distribution (Xiong \& Proctor, 2015) due to the 
Robot's social gaze affects conflict resolution

222 dissipation of the automatic activation of the spatial corresponding response, the maximum time allowed to 223 respond was $1000 \mathrm{~ms}$. After a response was made, or the maximum time allowed expired, a video depicting

224 iCub establishing Mutual or Avoiding gaze was presented for 6000 ms (see Video 1). The inter-trial interval

225 was $1000 \mathrm{~ms}$. Participants were also instructed to pay attention to the iCub following their response 226 because at the end of the experiment they would be asked to answer questions about its behaviour. At the 227 end of each block, feedback indicating the cumulative amount of correct responses, and the average 228 response time across the experiment was presented.

229 The task consisted of 4 blocks with 64 trials each. A short practice of 8 trials preceded the task. In each 230 block, the trial sequence was controlled so that each trial was preceded by either a corresponding (C) or 231 non-corresponding (NC) trial, with equal probabilities to be preceded by Avoiding or Mutual Gaze condition.

232 As a result, in both Avoiding and Mutual gaze conditions, four different trial sequences occurred (C-C, C$233 \mathrm{NC}, N C-\mathrm{C}, N C-\mathrm{NC}$, with italics denoting trial $n-1)$. << INSERT VIDEO 1 HERE: https:/www.youtube.com/watch?v=JWV7I4rutHA >>

\section{Data Analysis}

236 We excluded from analyses the first trial of each block, trials that were preceded by an incorrect response, 237 incorrect responses, and correct responses faster than $150 \mathrm{~ms}$ and slower than $1000 \mathrm{~ms}(7.0 \%$ of the 238 administered trials). Mean correct RTs were submitted to a repeated-measures analysis of variance 239 (ANOVA) with Trial $n-1$ Correspondence ( $C$ vs. $N C$ ), Trial $n$ Correspondence (C vs. NC), and Preceding 240 Gaze Feedback (Avoiding vs. Mutual) as within-subject factors. When necessary, comparisons were 241 performed using paired samples t-tests. Analysis was run using the Jasp software (v.0.9.2).

\section{Results and Discussion}

243 The analysis revealed a significant main effect of Trial $n$ Correspondence, $F(1,19)=156.74, p<.001, \eta_{p}^{2}$

$244=.89$, indicating faster responses for corresponding $(\mathrm{M}=464 \mathrm{~ms}, \mathrm{SE}=2.1 \mathrm{~ms})$ than for non-corresponding 245 trials $(\mathrm{M}=515 \mathrm{~ms}, \mathrm{SE}=2.2 \mathrm{~ms})$, indicating the classical SE. The correspondence effect was modulated by 246 correspondence sequence, as indicated by the significant interaction between Trial $\mathrm{n}$ Correspondence and 247 Trial $n-1$ Correspondence, $F(1,19)=14.18, p<.001, \eta_{p}^{2}=.43$. Planned comparisons showed a 59-ms SE 248 after a corresponding trial, $\mathrm{t}(19)=13.3, \mathrm{p}<.001, \mathrm{~d}=3.0$; and a SE of 28-ms after a non-corresponding trial, 
Robot's social gaze affects conflict resolution

$249 t(19)=8.9, p<.001, d=2.0$. Paired simple t-test showed that the two effects differed in magnitude, $t(19)=$ $2503.8, p<.001, d=.8$. No other main effect or interaction were significant, all ps $>.094$.

251 The results of Experiment 1 showed that the SE was modulated by the correspondence of the 252 preceding trial. Specifically, in line with previous studies, the typical trial-by-trial adaptations occurred (e.g., 253 Ciardo et al., 2019; lani et al., 2014) with a larger SE evident following a corresponding (59 ms) than a non254 corresponding trial $(28 \mathrm{~ms})$. These results support the idea that the detection of conflict in trial $n-1$ triggers 255 adaptations that are aimed at eliminating the impact of spatial S-R correspondence on response selection 256 in the following trial (Braver, 2012; Ridderinkhof, 2002). No effect of preceding gaze feedback emerged. 257 Such results may suggest that the mutual and avoiding gaze feedback of a humanoid robot may not act as 258 an affective signal and do not affect performance in the subsequent trial. However, in Experiment 1 mutual 259 and avoiding gaze feedbacks were manipulated through a robot face presented on the screen. Such a 260 setup may lack ecological validity. Indeed, several studies showed that embodiment plays a crucial role 261 when investigating social cognition mechanisms (e.g. Vasco et al., 2019; Wiese, Metta, Wykowska, 2017), 262 especially in the context of mutual gaze, with higher arousal and faster responses associated with real-time 263 mutual gaze compared to direct gaze presented on a screen (Hietanen et al., 2008; Pönkänen et al., 2011).

264 Taking this into account, we designed Experiment 2, in which we implemented the screen-based paradigm 265 to a 3-D physical-presence setup in which the mutual and avoiding gaze feedbacks from iCub were 266 displayed in real-time.

\section{Experiment 2}

268 Materials and Methods

269 Participants.

270 Twenty-five new participants (7 males; 3 lefthanded; Mean age: $25.6 \pm 4.2$ years) took part in the study. All 271 participants had normal or corrected-to-normal vision and were not informed about the purpose of the 272 experiment. All gave their informed consent before participating and the study was conducted under the 273 same ethical procedures and protocol as in Experiment 1. The data of five participants were excluded due 274 to: technical failure of the robot (2), technical failure of the program (2), or because the participant was 275 unable to perform the task (1). Therefore, data of twenty participants were further analyzed. 
Robot's social gaze affects conflict resolution

Apparatus and Stimuli.

277 The experiment was carried out in a dimly lit and noiseless room. The participant was seated facing the

278 iCub robot at a viewing distance of $70-80 \mathrm{~cm}$ (see Figure 1). We used a version of the iCub robot consisting

279 of a full robotic head and a 3D printed torso mounted on a stool. The iCub's eyes have 3 degrees of freedom

280 (common tilt, vergence, and version) and three additional degrees of freedom in the neck (roll, pitch, yaw).

281 To control the eyes and the neck of iCub, we used the YARP (Yet Another Robot Platform, Metta, Fitzpatrick,

$282 \&$ Natale, 2006) Python wrappers.

283 In our procedure, to make iCub gaze at a specific location, a 6-DOF gaze controller has been used

284 (Roncone, Pattacini, Metta, \& Natale, 2016). This controller uses inverse kinematics to find eyes and neck

285 positions to make the robot look at certain 3D Cartesian coordinates. In our experiment, the target location

286 was predefined for the Avoiding gaze feedback $\left(x: 0^{\circ}, y:-26^{\circ}, z: 3^{\circ}\right)$, whereas, in the Mutual gaze feedback

287 coordinates of target location were calculated online in each trial, to make the robot establish eye contact

288 in real-time (see Video 2). Participant's eyes were detected using a face detection algorithm applied to the

289 images coming from the camera sensors placed in the eye bulbs of the iCub robot (Cao, Hidalgo Martinez,

290 Simon, Wei, Sheikh, 2017). The vergence of the eyes was set to 3 degrees and maintained constant. The

291 trajectory time for the movement of eyes and neck was set to $500 \mathrm{~ms}$, to maintain the impression of a

292 smooth and naturalistic movement.

293 Stimuli were the same as those used in Experiment 1. Responses were executed as in Experiment 1. The

294 keyboard was placed on the participant's lap centrally with respect to the body midline. Stimulus

295 presentation, response timing, and data collection were controlled as in Experiment 1.

296 < INSERT VIDEO 2 HERE: https://www.youtube.com/watch?v=eOktX7GCuJA >>

297 Procedure.

298 As in Experiment 1, participants were instructed to discriminate the tone pitch in one of the headphones,

299 ignoring its spatial location. Participants were also instructed to pay attention to the iCub because at the

300 end of the experiment they would be asked to answer questions about its behaviour. Each trial started with

301 the robot depicting a neutral expression and closed eyes with the neck located at the rest position. Following

$3021500 \mathrm{~ms}$ the expression of the robot turned from neutral to surprise for $1700 \mathrm{~ms}$. $200 \mathrm{~ms}$ after the facial 
Robot's social gaze affects conflict resolution

303

\section{7}

expression changed the stimulus tone was played. The maximum time allowed to respond was set at 1000 ms from stimulus onset. After a response was given, or the allowed response time elapsed, the robot opened the eyes and moved the neck and the eyes toward the Avoiding or Mutual gaze target location, see Video 2. At the end of each block, feedback indicating the cumulative amount of correct responses and the average response time across the task was given to participants. Contrary to Experiment 1, instructions and feedback were given verbally by iCub using the text to speech library: https://github.com/robotology/speech/tree/master/svox-speech. The task consisted of 5 blocks of 48 trials each. A short practice of 8 practice trials preceded the task. As in Experiment 1, in each block, the trial sequence was controlled so that each trial was preceded by either a corresponding (C) or noncorresponding (NC) trial, with equal probabilities to be preceded by Avoiding or Mutual Gaze condition. As a result in both Avoiding and Mutual gaze conditions, four different trial sequences occurred $(C-C, C-N C$, $N C-C, N C-N C$, with italics denoting trial $n-1)$.

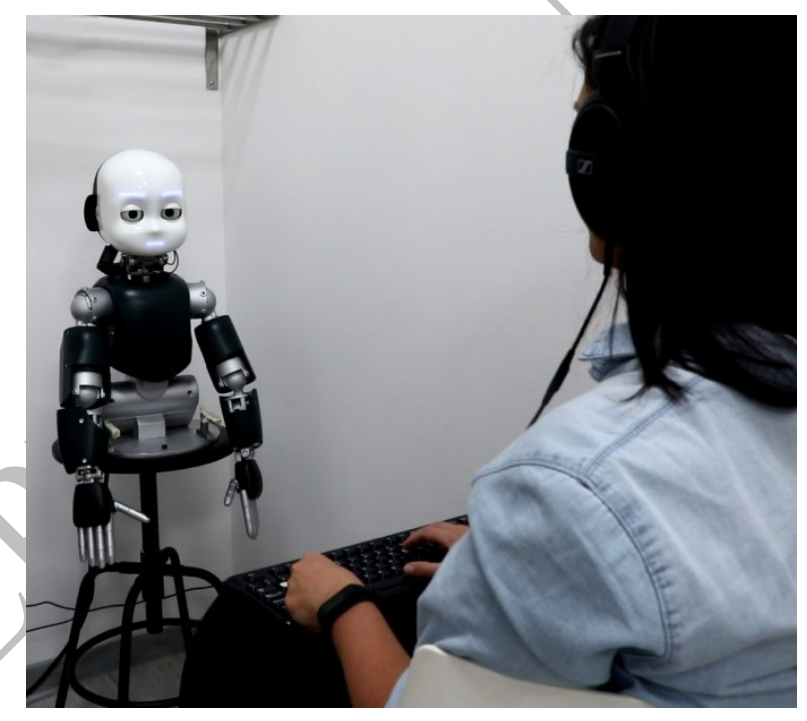

Figure 1 Experimental setup in Experiment 2.

\section{Data Analysis}

As for Experiment 1, the first trial of each block, trials that were preceded by an incorrect response, incorrect responses, and correct responses faster than $150 \mathrm{~ms}$ and slower than $1000 \mathrm{~ms}$ were excluded from the analysis (12.0\% of the administered trials). Mean correct RTs were submitted to a repeated-measures analysis of variance (ANOVA) with Trial $n-1$ Correspondence (C vs. NC), Trial $n$ Correspondence (C vs. 
Robot's social gaze affects conflict resolution

322 NC), and Preceding Gaze Feedback (Avoiding vs. Mutual) as within-subject factors. When necessary, 323 comparisons were performed using paired samples t-tests.

\section{Results and Discussion}

325 The analysis on RTs showed that responses were faster following Avoiding gaze $(\mathrm{M}=456 \mathrm{~ms}, \mathrm{SE}=1.9$ $326 \mathrm{~ms})$ than Mutual gaze trials $(\mathrm{M}=472 \mathrm{~ms}, \mathrm{SE}=2.2 \mathrm{~ms})$, as indicated by the main effect of Preceding Gaze, $327 F(1,19)=69.67, p<.001, \eta_{p}^{2}=.79$. The main effect of Trial $n$ Correspondence was significant, $F(1,19)=$ $32869.67, p<.001, \eta_{p}^{2}=.79$, with faster responses for corresponding (M = $\left.442 \mathrm{~ms}, \mathrm{SE}=2.1 \mathrm{~ms}\right)$ than for non329 corresponding trials $(\mathrm{M}=485 \mathrm{~ms}, \mathrm{SE}=1.9 \mathrm{~ms})$, indicating a classical SE. This correspondence effect was

330 modulated by correspondence sequence, as indicated by the significant interaction between Trial $n$ 331 Correspondence and Trial $n-1$ Correspondence, $F(1,19)=22.23, p<.001, \eta_{p}^{2}=.54$. Planned comparisons 332 showed the typical sequential effects reported in Experiment 1 and in previous studies (e.g., Ciardo et al., 333 2019; lani et al., 2014) with a 59-ms SE after corresponding trials, $t(19)=8.8, p<.001, d=2.0$; and a 43 $334 \mathrm{~ms}$ SE after a non-corresponding trials, $\mathrm{t}(19)=4.9, \mathrm{p}<.001, \mathrm{~d}=1$.1. Paired-samples $\mathrm{t}$-test showed that 335 the two effects differed in magnitude, $t(19)=4.72, p<.001, d=1.05$. Importantly, the two-way interaction 336 between Preceding Gaze Feedback and Trial $n$ Correspondence was significant, $F(1,19)=6.35, p=.021$, $337 \eta_{p}^{2}=.25$. Planned comparison showed that a larger SE occurred following Mutual gaze feedback (51 ms) 338 than Avoiding gaze feedback (35 ms), $t(19)=2.59, p=.021, d=.56$ (see Figure 2). No other main effects 339 or interactions were significant, all ps $>.14$.

340 Table 1. Mean correct reaction times and standard deviation (in milliseconds) as a function of Experiment 341 (Exp1 vs Exp2), Preceding Gaze Feedback (Avoiding vs Mutual), Trial $n-1$ (corresponding, $C$ vs non342 corresponding, NC), and Trial $n$ (corresponding, $C$ vs non-corresponding, NC).

\begin{tabular}{|c|c|c|c|c|c|}
\hline 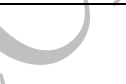 & $\begin{array}{l}\text { Preceding Gaze } \\
\text { Feedback }\end{array}$ & Trial n-1 & Trial $\mathrm{n}$ & Mean & SD \\
\hline \multirow{8}{*}{ Exp.1 } & \multirow{4}{*}{ Avoiding } & \multirow{2}{*}{ C } & $C$ & 450 & 89 \\
\hline & & & $\mathrm{NC}$ & 509 & 106 \\
\hline & & \multirow{2}{*}{ NC } & C & 471 & 96 \\
\hline & & & $\mathrm{NC}$ & 515 & 98 \\
\hline & \multirow{4}{*}{ Mutual } & \multirow{2}{*}{ C } & C & 461 & 94 \\
\hline & & & $\mathrm{NC}$ & 520 & 96 \\
\hline & & \multirow{2}{*}{$\mathrm{NC}$} & C & 474 & 95 \\
\hline & & & NC & 516 & 103 \\
\hline
\end{tabular}


Robot's social gaze affects conflict resolution

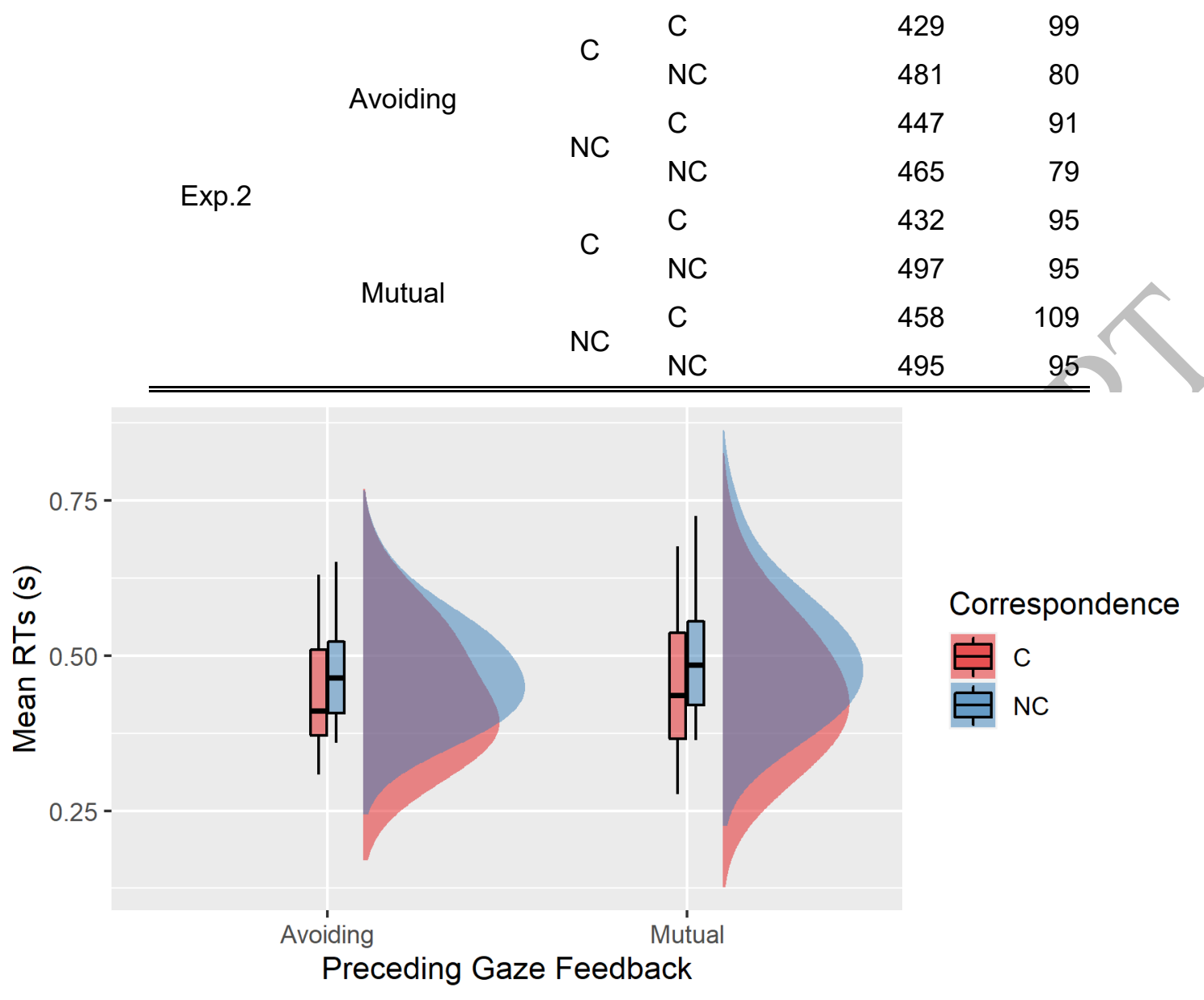

Figure 2 Mean reaction times in Experiment 2 for the corresponding (C) and non-corresponding (NC) Trial n, as a 345 function of Preceding Gaze Feedback (Avoiding vs Mutual).

346 Results of Experiment 2 showed that mutual and avoiding gaze feedback differently affected the SE

347 in the subsequent trial. Specifically, we found that the SE was smaller in magnitude following avoiding gaze

348 feedback, relative to mutual gaze (35 vs. 51, respectively). Social gaze feedback did not modulate

349 sequential effects, as there was no significant three-way interaction. These results indicate that social gaze

350 feedback modulated conflict within a single trial, but not adaptation across trials. Hence, it seems that the

351 ability to manage conflict within a single trial and the ability to adapt performance based on the conflict

352 experienced in the previous trial depend on different mechanisms. Only the first mechanism appears to be

353 sensitive to the social gaze feedback delivered by the robot. Given that our experimental design did not

354 include a neutral condition, we cannot conclude the direction of our manipulation on the magnitude of the

355 SE. To estimate the direction of the SE in the two critical conditions of our protocol (mutual vs. avoiding 
Robot's social gaze affects conflict resolution

356 gaze feedback), we ran a follow-up control experiment (Exp3) in which the robot was not providing any 357 feedback to the participants.

\section{$358 \quad$ Experiment 3}

359 Materials and Methods

360 Participants.

361 Twenty-five new participants (6 males; 1 lefthanded; Mean age: $29.2 \pm 11.4$ years) took part in the study.

362 All participants had normal or corrected-to-normal vision and were not informed about the purpose of the

363 experiment. All gave their informed consent before participating and the study was conducted under the 364 same ethical procedures and protocol as in Experiment 1. The data of five participants were excluded due 365 to: Age of the participant above 45 years old (2 participants, see Van der Lubbe \& Verleger, 2002), technical 366 failure of the program (1 participant), because the participant committed too many errors (1 participant; 367 error rate: $18 \%$ ), or because outliers removal reduced to less than $50 \%$ the trials to be included in the 368 analysis (1 participant). Data of twenty participants were further analyzed.

369 Apparatus, Stimuli, and Procedure.

370 The apparatus, stimuli, and response execution were the same of Experiment 2. Stimulus presentation, 371 response timing, and data collection were controlled as in Experiment 2.

372 The procedure was the same as Experiment 2, with the only exception that after a response was given, or 373 the allowed response time elapsed, the robot did not open the eyes and did not move the neck and the 374 eyes. At the end of each block, feedback indicating the cumulative amount of correct responses and the 375 average response time across the task was given to participants. As in Experiment 2, instructions and 376 feedback were given verbally by iCub. The task consisted of 3 blocks of 40 trials each. A short practice of 3778 practice trials preceded the task. Since we were not interested anymore in sequential adaptations, the 378 trial presentation order was fully randomized.

379 Data Analysis 
Robot's social gaze affects conflict resolution

As in Experiment 2, the first trial of each block, trials that were preceded by an incorrect response, incorrect responses, and correct responses faster than $150 \mathrm{~ms}$ and slower than $1000 \mathrm{~ms}$ were excluded from the analysis (9.2\% of the administered trials). Mean correct RTs for corresponding and non-corresponding trials

383 were compared using paired samples t-tests.

\section{Results}

385 The analysis on RTs showed that responses were faster for corresponding ( $\mathrm{M}=420 \mathrm{~ms}$, SE $=1.7 \mathrm{~ms})$ than 386 for non-corresponding trials $(M=454 \mathrm{~ms}, \mathrm{SE}=1.7 \mathrm{~ms}), \mathrm{t}(19)=4.83, p<.001, d=1.08$.

Results of Experiment 3 showed that when participants were not provided with any feedback from 388 the robot, our setup induced a Simon effect of $34 \mathrm{~ms}$.

\section{The role of embodiment: Comparison between Exp1 and Exp2.}

390 To assess the effect of embodiment, we conducted an additional analysis to compare the data of the two 391 experiments. The IQR method on the average RTs of correct trials for each participant was applied to 392 identify possible outliers. Indeed, 1 participant showed an average RT above the upper limit (Q3+1.5 IQR).

393 Thus we ran the analysis excluding this participant on a sample size of $\mathrm{N}=39$. Mean $\mathrm{RT}$ s were entered into 394 an ANOVA with Trial n Correspondence (C vs. NC) and Preceding Gaze Feedback (Avoiding vs. Mutual) 395 as within-subject factors and Experiment (Exp1 vs.Exp2) as a between-subjects factor. In addition to the 396 main effect of Trial $n$ Correspondence, $F(1,37)=209.67, p<.001, \eta_{p}^{2}=.85$, the analysis revealed a main 397 effect of Preceding Gaze, $F(1,37)=5.87, p=.020, \eta_{p}^{2}=.14$ and a significant two-way interaction Trial $n$ 398 Correspondence * Preceding Gaze Feedback, $F(1,37)=4.90, p=.033, \eta_{p}^{2}=.12$. Importantly, the three399 way interaction was significant, $F(1,37)=5.53, p=.039, \eta_{p}^{2}=.11$. Independent T-test showed a marginally 400 significant difference in the magnitude of the $S E, t(37)=2.0, p=.056, d=.63$ (see Figure 3). No significant 401 difference in the SE occurred across experiments following a Mutual gaze, $t<1$. No other main effects or 402 interactions were significant, all ps > .326. 


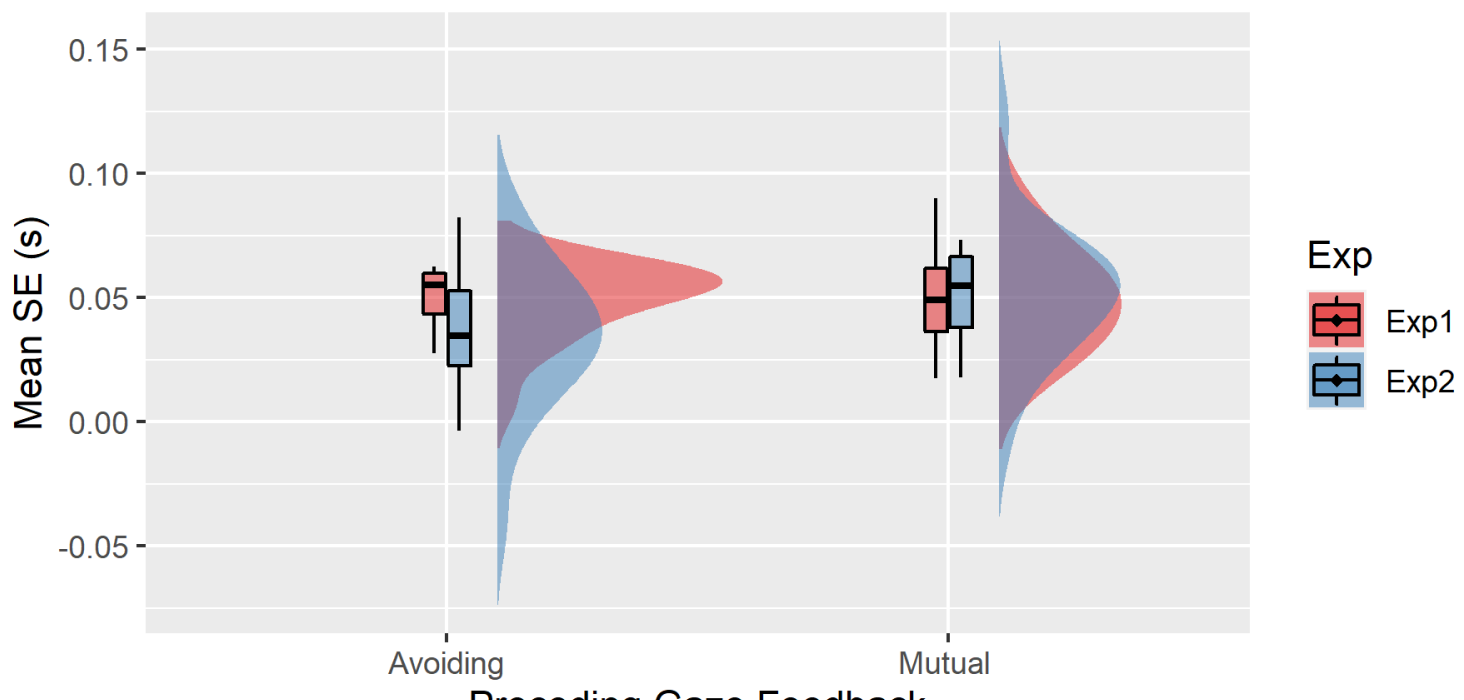

403

404

405

406

407

408 $420=0.58$.

Preceding Gaze Feedback

Figure 3. Mean Simon Effect (s) plotted as a function Preceding Gaze Feedback (Avoiding vs. Mutual) across experiments.

\section{The effect of social gaze: Comparison between Exp2 and Exp3.}

We ran two exploratory analyses to compare the SE elicited following Mutual and Avoiding gaze feedback with the SE when no feedback was provided. Specifically, we compared the SE of Exp 3 with the

SE of Exp2 for mutual gaze and avoiding gaze conditions separately. Mean RTs were entered into an ANOVA with Trial n Correspondence (C vs. NC) as within-subject factors and Gaze Condition (Avoiding or Mutual vs. Neutral) as a between-subjects factor.

Avoiding vs Neutral

Apart from the main effect of Trial $n$ Correspondence, $F(1,38)=51.70, p<.001, \eta_{p}^{2}=.585$, no other main effects or interactions were significant, all ps $>.470$.

\section{Mutual vs Neutral}

The analysis showed a main effect of Trial $n$ Correspondence, $F(1,38)=87.22, p<.001, \eta_{p}^{2}=.70$. The two-way interaction Trial n Correspondence ${ }^{*}$ Gaze Condition showed a tendency to a significant effect, $F(1$, $38)=3.39, p=.073, \eta_{p}^{2}=.08$. Independent-samples t-test showed that the 34-ms SE of the Neutral gaze

419 condition marginally differed from the 51-ms SE following mutual gaze feedback, $t(38)=1.84, p=.073, d$ 


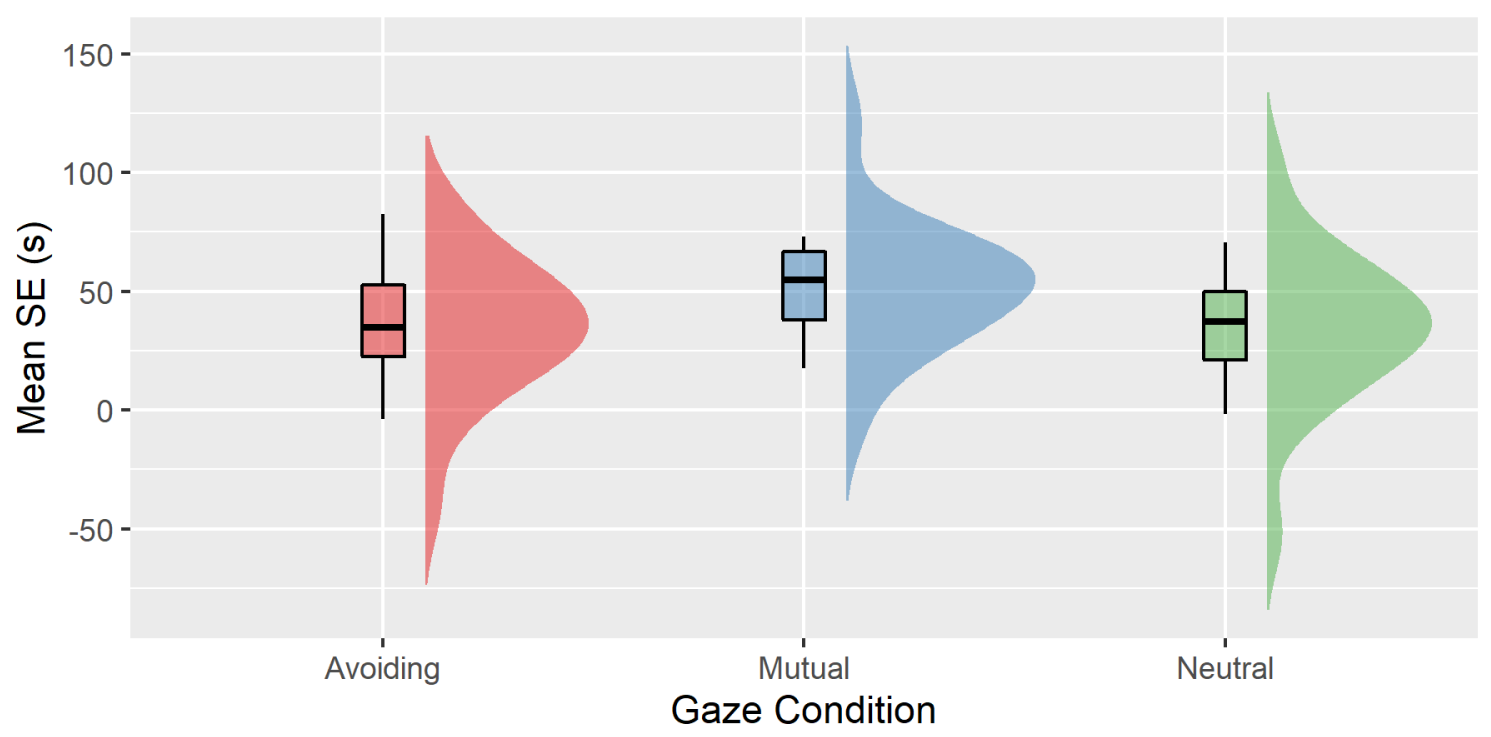

422 Figure 4. Mean Simon Effect (s) plotted as a function Gaze Condition (Avoiding vs. Mutual vs. Neutral).

\section{General Discussion}

424 The main goal of the present study was threefold: i) investigating whether the social gaze feedback

425 of a robot can modulate cognitive control; ii) understanding if the social gaze feedback of a robot affects

426 cognitive control through within-trial conflict resolution (i.e., adjusting task parameters and priority settings

427 before the occurrence of cognitively demanding events) or it acts through trial-by-trial adaptations; iii) testing

428 the impact of the embodied physical presence of an agent displaying social gaze. To meet these aims, in

429 three experiments we evaluated both the SE and trial-by-trial adaptations as a function of the type of

430 feedback participants received in the previous trial from the iCub robot, namely, mutual or avoiding gaze.

431 Across experiments, we compared the effect of the social gaze feedback between screen-based (Exp1)

432 and embodied setups with the robot's physical presence (Exp2 and Exp3).

433 Results of Experiment 1 revealed that the 51 ms SE was modulated by the correspondence of the preceding

434 trial. Specifically, in line with previous studies, the typical sequential effects occurred (e.g., Ciardo et al.,

435 2019; lani et al., 2014; 2016) with a larger SE evident following a corresponding (59 ms) than a non-

436 corresponding trial $(28 \mathrm{~ms})$. Moreover, Experiment 1 showed that when the social gaze feedback was

437 manipulated in a screen-based setup, it did not modulate either conflict resolution or conflict adaptations.

438 In Experiment 2, apart from the expected correspondence and trial-by-trial adaptation effects, the results 
Robot's social gaze affects conflict resolution

439 showed faster responses following avoiding gaze than mutual gaze feedback. Most importantly for the aim 440 of the present study, results of Experiment 2 showed that when the social gaze feedback of the robot was 441 manipulated in a setup involving physical embodied presence, the SE was larger in magnitude following 442 mutual gaze feedback $(51 \mathrm{~ms})$ than following avoiding gaze $(35 \mathrm{~ms})$. No effect of social gaze emerged for 443 trial-by-trial adaptations as indicated by non-significant three-way interaction. Given that Experiment 2 did 444 not include a no-gaze condition, we ran the control Experiment 3 to estimate the baseline magnitude of the 445 SE elicited by our embodied setup.

446 Our results showed that when the robot did not display social gaze feedback, a SE of 34 ms was observed, 447 suggesting that our setup elicited overall a SE similar in magnitude to those reported in previous screen448 based studies using a similar procedure (e.g. D'ascenzo et al., 2018; Xiong \& Proctor, 2015). Such result 449 is not in line with previous evidence showing that conflict effects, such as the Stroop effect, are reduced in 450 the presence of another agent (Huguet et al.,1999; Spatola et al ., 2018; see Belletier et al 2019 for a 451 review).

452 Overall, our results suggest that mutual gaze feedback affects conflict resolution in the subsequent 453 trial but only with the physical presence of the robot, i.e, when the gaze behaviour can be established in 454 real-time.

455 Our results extend earlier findings showing for the first time that social gaze feedback (even when displayed by an artificial agent such as a humanoid robot) affects cognitive control. Specifically, its 457 modulatory effect on cognitive conflict appears to be in contrast to previous studies showing that the 458 negative affective state induced by losses or negative emotion enhances cognitive control resulting in a 459 smaller interference effect in conflict tasks (see van Steenbergen, 2015 for a review) and do not support 460 the affective-congruent hypothesis. Our results suggest that following mutual gaze feedback, conflict 461 resolution might be reduced in efficiency. Such a result is in line with evidence showing that mutual, but 462 not avoiding, gaze interferes with the inhibition of task-irrelevant information (Kompatsiari et al., 2021a). 463 Specifically, in a series of recent studies, Kompatsiari and colleagues showed that mutual gaze delays 464 attention disengagement from the non-predictive cue in a gaze cueing task. As a result, the distracting 465 effect of gaze cues was prolonged in time even when the stimulus onset asynchrony was equal to $1 \mathrm{~s}$ 466 (Kompatsiari et al., 2021b). Using a decision-making task Belkaid et al., (2021) showed that mutual gaze 
Robot's social gaze affects conflict resolution

467 delayed reaction times by increasing the decision threshold. Also, iCub's mutual gaze was associated with 468 higher alpha synchronization in the EEG signal, indicating that during mutual gaze with the robot, 469 suppression mechanisms were activated. In a similar vein, in our study, mutual gaze may have affected 470 attention control on response selection. As a consequence, participants were delayed in solving the 471 response conflict elicited by the task-irrelevant spatial feature in non-corresponding trials. Interestingly, our 472 results showed that the effect of mutual gaze occurs not only when social gaze is provided as a cue 473 (Kompatsiari et al., 2021b) but also when the social gaze is manipulated as feedback, as its effect was 474 transferred to the processing of a stimulus presented in a subsequent trial. Our results can be considered 475 in the context of the proposal that social brain networks may be in antagonism with the frontoparietal 476 attentional network (e.g. Bossi et al., 2020; Anticevic et al., 2012; Fox et al., 2005). In line with this reasoning, 477 it could be that when presented with a socially relevant event, like the communicative mutual gaze, the 478 recruitment of social brain areas may act against the attentional focus. Future studies should systematically 479 address this hypothesis both at the neural and behavioural levels. One intriguing issue that remains to be 480 addressed is whether social signals interfere at the target processing level or the response selection stage. 481 This could be examined by presenting social gaze at the same time as the target stimulus.

482 Overall, our results indicate that social gaze feedbacks of a robot seem to be effective in modulating conflict 483 resolution but not for conflict adaptions. A similar dissociation has been shown in literature in those studies 484 that investigated individual differences in the cognitive control mechanism. For instance, lani et al. (2014) 485 showed that while conflict resolution in a given trial is affected by age of the participants, the trial-by-trial 486 adaptations are not. Specifically, the authors reported a larger SE for first-grade children (6-7 years) 487 compared to second-graders (7-8 years) children, however, the reduction in the magnitude of the SE 488 following a conflict event (i.e. a non-corresponding trial) was comparable across groups (see also Larson, 489 et al., 2012; for similar results in elderly).

490 The lack of modulation in the trial-by-trial adaptations could be due to the fact that in our study, iCub's gaze 491 feedback was independent of participants' performance. Strümer and colleagues (2011) showed that, in a 492 Simon task, monetary gains and losses in between trials affected trial-by-trial adaptations only when they 493 were contingent on the actual performance (i.e., reward and penalties were assigned to the $25 \%$ fastest 494 and slowest responses) but not when they occurred randomly. Similar results have been reported also 
Robot's social gaze affects conflict resolution

495 using different conflict tasks, such as the Flanker task and the task-switch paradigm (Braem et al., 2012).

496 Future studies should investigate the effect of the social gaze feedbacks of a robot in affecting conflict

497 resolution and conflict adaptations in a response-contingent way. For instance, mutual and avoiding gaze

498 feedbacks could be provided to reward or punish the $25 \%$ fastest and slowest responses.

Finally, by comparing the effect of the social gaze feedback between screen-based and embodied

500 setups, our results show that only the latter was effective in affecting conflict resolution. Such a result

501 extends previous evidence showing that when investigating social gaze, screen-based protocols might lack

502 ecological validity (Kompatsiari et al., 2021b). For instance, Hietanen et al., (2008) showed that real-time

503 mutual gaze was associated with greater sympathetic arousal (skin conductance responses), than averted

504 gaze. Similarly, Pönkänen et al. (2011) reported different EEG asymmetries for faces that were presented

505 live through an electronic shutter and those that were presented as pictures on a computer screen. It has

506 been proposed that real-time social gaze is likely to play a greater role in influencing sensations of intimacy,

507 experienced self-relevance, and awareness about being seen by another person (or a robot) who is

508 physically present, as compared to seeing a picture of a face on a computer screen (Hietanen et al.,

509 2008, Pönkänen et al., 2011; Kompatsiari et al., 2018; 2019).

510 We believe that our results are crucial not only for fundamental research but also for applied

511 scenarios. The findings open up the possibility of using robots to train complex cognitive skills like self-

512 regulation. The main advantage of embedding robots within cognitive training protocols is the possibility of

513 maintaining good experimental control on the one hand and increased ecological validity on the other, an

514 aspect crucial for certain mechanisms of cognition, as our data show. Through their social presence, robots

515 can interact in real-time with the user in a shared environment. Through the implementation of motivational

516 and social behaviours, we believe that robots can improve learning. To date, robots have been mostly used

517 in rehabilitation protocols for motor and social skills, such as joint attention (see Chevalier et al., 2019 for a

518 review). However, taking into consideration that cognitive control, and response inhibition specifically, is

519 impaired not only in aging but also in several psychiatric disorders, such as but not limited to, eating and

520 mood disorders, Tourettes' syndrome, and obsessive-compulsive spectrum disorders, we propose that also

521 cognitive control training protocols can benefit from the implementation of robots. For instance, several

522 studies showed that children with attention-deficit/hyperactivity disorder (ADHD) report larger Simon and 
Robot's social gaze affects conflict resolution

523 Flanker effects when compared to children with typical development (for a review see Mullane, Corkum, 524 Klein, \& McLaughlin, 2009). Also, it has been shown that conflict resolution and conflict adaptation 525 mechanisms are differently impaired in patients with eating disorders (Bartholdy et al., 2017). Using a stop526 signal task Bartholdy and colleagues showed that conflict resolution was enhanced in women with anorexia 527 compared to healthy controls, whereas no difference across groups was reported for reactive control 528 mechanisms. Similarly, Sellaro and Colzato (2017) reported a larger SE for overweight individuals 529 compared to normal-weight controls.

530 To conclude, the present study shows that robots' social gaze feedback modulates conflict resolution 531 in a Simon task but not conflict adaptations. The modulatory effect was observed for the embodied setup 532 in which the robot could engage or avoid eye contact in real-time, opening up the way to a new potential 533 application of robots in protocols aiming to investigate, and train, cognitive control and self-regulation in 534 both healthy and clinical populations. 
Robot's social gaze affects conflict resolution

\section{Acknowledgments}

This work received support by the European Research Council (ERC) under the European Union's

537 Horizon 2020 research and innovation program (grant awarded to AW, titled "InStance: Intentional Stance

538 for Social Attunement." G.A. No: ERC-2016-StG-715058). The content of this paper is the sole responsibility

539 of the authors. The European Commission or its services cannot be held responsible for any use that may

540 be made of the information it contains

541 We are thankful to Dr. De Tommaso for having programmed the iCub robot. 
Robot's social gaze affects conflict resolution

\section{Additional information}

543 Data, videos, and stimuli are available at https://osf.io/nvcz6/

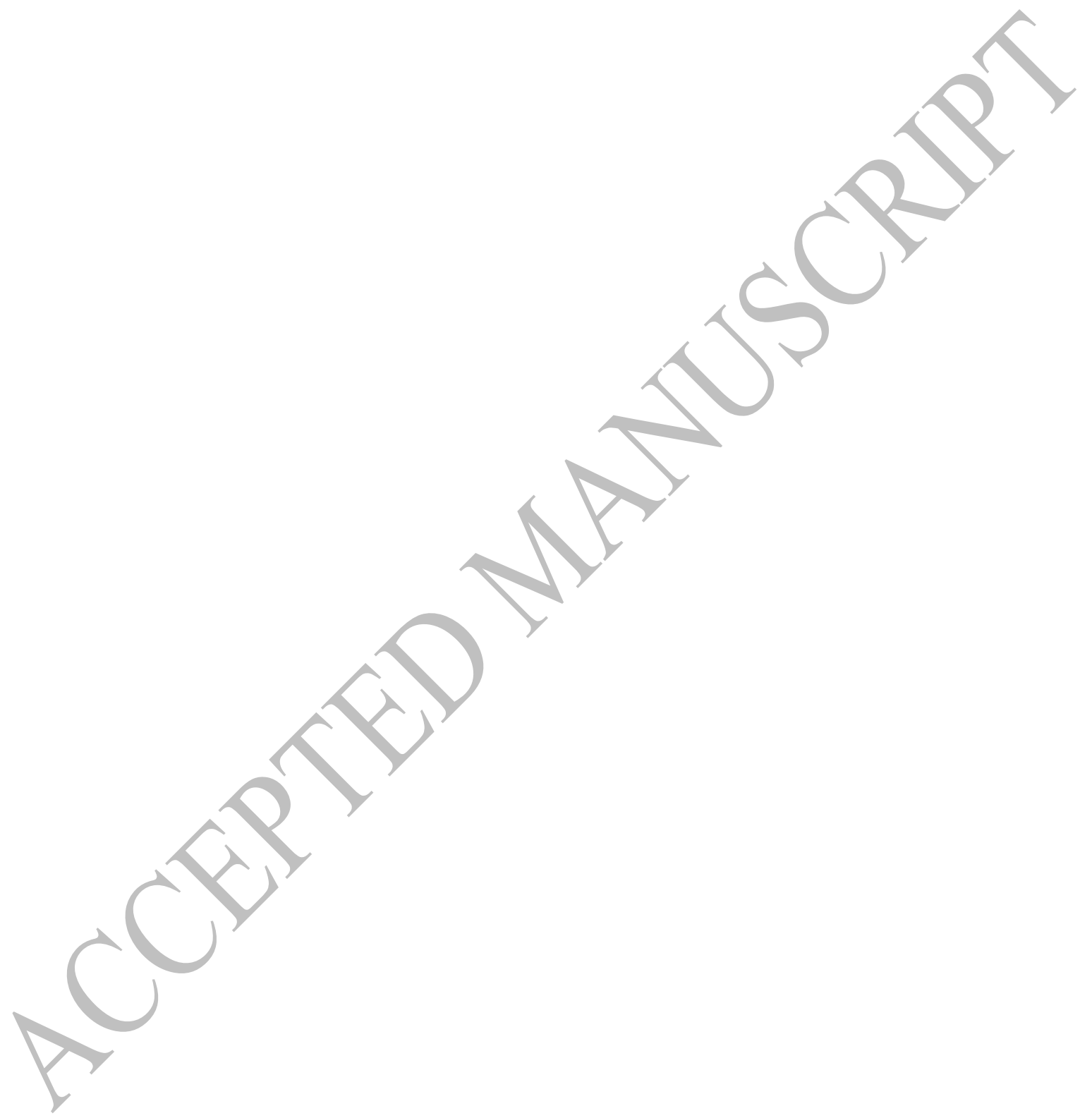


Robot's social gaze affects conflict resolution

544

545

546

547

548

549

550

551

552

553

554

555

556

557

558

559

560

561

562

563

564

565

566

567

568

569

570

571

572

573

574

575

576

577

\section{References}

Anticevic, A., Cole, M. W., Murray, J. D., Corlett, P. R., Wang, X. J., \& Krystal, J. H. (2012). The role of default network deactivation in cognition and disease. Trends in cognitive sciences, 16(12), 584-592.

Bartholdy, S., Rennalls, S. J., Jacques, C., Danby, H., Campbell, I. C., Schmidt, U., \& O'Daly, O. G. (2017). Proactive and reactive inhibitory control in eating disorders. Psychiatry Research, 255, 432-440.

Belkaid, M., Kompatsiari, K., de Tommaso D., Zablith I., Wykowska A. (2021). Mutual gaze with a robot affects human neural activity and delays decision-making processes. Science Robotics, 6 (58). doi:10.1126/scirobotics.abc5044

Belletier, C., Normand, A., \& Huguet, P. (2019). Social-facilitation-and-impairment effects: From motivation to cognition and the social brain. Current Directions in Psychological Science, 28(3), 260-265.

Bossi, F., Willemse, C., Cavazza, J., Marchesi, S., Murino, V., \& Wykowska, A. (2020). The human brain reveals resting state activity patterns that are predictive of biases in attitudes toward robots. Science robotics, 5(46).

Botvinick, M. M. (2007). Conflict monitoring and decision making: Reconciling two perspectives on anterior cingulate function. Cognitive Affective \& Behavioural Neuroscience, 7, 356-366. doi: 10.3758/CABN.7.4.356.

Botvinick, M. M., Braver, T. S., Barch, D. M., Carter, C. S., \& Cohen, J. D. (2001). Conflict monitoring and cognitive control. Psychological Review, 108, 624-652.

Braem, S., Verguts, T., Roggeman, C., \& Notebaert, W. (2012). Reward modulates adaptations to conflict. Cognition, 125, 324-332. doi: 10.1016/j.cognition.2012.07.015.

Braver, T. S. (2012). The variable nature of cognitive control: A dual mechanisms framework. Trends in Cognitive Sciences, 16, 106-113. doi:10.1016/j.tics.2011.12.010

Cabanac, M. (1992). Pleasure: The common currency. Journal of Theoretical Biology, 155, 173-200. doi: 10.1016/S0022-5193(05)80594-6.

Cao, Z., Simon, T., Wei, S. E., \& Sheikh, Y. (2017). Realtime multi-person 2d pose estimation using part affinity fields. In Proceedings of the IEEE conference on computer vision and pattern recognition (pp. 7291-7299).

Chevalier, P., Kompatsiari, K., Ciardo, F., \& Wykowska, A. (2019). Examining joint attention with the use of humanoid robots-A new approach to study fundamental mechanisms of social cognition. Psychonomic Bulletin \& Review, 1-20.

Ciardo, F., Beyer, F., De Tommaso, D., \& Wykowska, A. (2020). Attribution of intentional agency towards robots reduces one's own sense of agency. Cognition, 194, 104109.

Ciardo, F., \& Wykowska, A. (2020). La robotica assistenziale sociale come strumento per promuovere lo sviluppo socio-cognitivo: vantaggi, limiti e prospettive future. Sistemi intelligenti, 32(1), 9-25. 
Robot's social gaze affects conflict resolution

578

579

580

581

582

583

584

585

586

587

588

589

590

591

592

593

594

595

596

597

598

599

600

601

602

603

604

605

606

607

608

609

Ciardo, F., Ricciardelli, P., \& lani, C. (2019). Trial-by-trial modulations in the orienting of attention elicited by gaze and arrow cues. Quarterly Journal of Experimental Psychology, 72(3), 543-556.

Cole, G. G., Smith, D. T., \& Atkinson, M. A. (2015). Mental state attribution and the gaze cueing effect. Attention, Perception, \& Psychophysics, 77(4), 1105-1115.

D’Ascenzo, S., Lugli, L., Baroni, G., Guidotti, R., Rubichi, S., lani, C., \& Nicoletti, R. (2018). Visual versus auditory Simon effect: A behavioural and physiological investigation. Quarterly Journal of Experimental Psychology, 71(4), 917-930.

De Jong, R., Liang, C.-C., \& Lauber, E. (1994). Conditional and unconditional automaticity: A dual-process model of effects of spatial stimulus-response correspondence. Journal of Experimental Psychology: Human Perception and Performance, 20, 731-750. doi:10.1037/0096- 1523.20.4.731

Dignath, D., Eder, A. B., Steinhauser, M., \& Kiesel, A. (2020). Conflict monitoring and the affective-signaling hypothesis-An integrative review. Psychonomic Bulletin \& Review, 27(2), 193-216.

Egner, T., \& Hirsch, J. (2005). Cognitive control mechanisms resolve conflict through cortical amplification of task-relevant information. Nature Neuroscience, 8, 1784-1790. doi:10.1038/nn1594

Egner, T., Ely, S., \& Grinband, J. (2010). Going, going, gone: characterizing the time-course of congruency sequence effects. Frontiers in psychology, 1, 154.

Emery, N. J. (2000). The eyes have it: the neuroethology, function and evolution of social gaze. Neuroscience \& biobehavioural reviews, 24(6), 581-604.

Eriksen, B. A., \& Eriksen, C. W. (1974). Effects of noise letters upon the identification of a target letter in a nonsearch task. Perception \& psychophysics, 16, 143-149.

Fox, M. D., Snyder, A. Z., Vincent, J. L., Corbetta, M., Van Essen, D. C., \& Raichle, M. E. (2005). The human brain is intrinsically organized into dynamic, anticorrelated functional networks. Proceedings of the National Academy of Sciences, 102(27), 9673-9678.

Hietanen, J. K., Leppänen, J. M., Peltola, M. J., Linna-aho, K., \& Ruuhiala, H. J. (2008). Seeing direct and averted gaze activates the approach-avoidance motivational brain systems. Neuropsychologia, 46(9), 2423-2430.

Hinz, N. A., Ciardo, F., \& Wykowska, A. (2021). ERP markers of action planning and outcome monitoring in human-robot interaction. Acta Psychologica, 212, 103216.

Hommel, B., Müsseler, J., Aschersleben, G., \& Prinz, W. (2001). The theory of event coding (TEC): A framework for perception and action planning. Behavioural and brain sciences, 24(5), 849.

Hommel, B., Proctor, R. W., \& Vu, K. P. L. (2004). A feature integration account of sequential effects in the Simon task. Psychological Research, 68, 1-17. doi: 10.1007/s00426- 003-0132-y 
Robot's social gaze affects conflict resolution

610

Huguet, P., Galvaing, M. P., Monteil, J. M., \& Dumas, F. (1999). Social presence effects in the Stroop task: further evidence for an attentional view of social facilitation. Journal of personality and social psychology, 77(5), 1011.

Humphrey, N. K. (1976). The social function of intellect. In Growing points in ethology, Cambridge: Cambridge University Press, pp. 303-317.

lani, C., Ciardo, F., Ricciardelli, P., \& Nicoletti, R. (2016). Relazione tra campo dipendenza-indipendenza e controllo cognitivo. Giornale italiano di psicologia, 43(3), 649-658.

Iani, C., Stella, G., \& Rubichi, S. (2014.). Response inhibition and adaptations to response conflict in 6-to 8-year-old children: Evidence from the Simon effect. Attention, Perception \& Psychophysics, 76, 12341241. doi:10.3758/s13414-014-

Kanske, P., \& Kotz, S. A. (2010). Modulation of early conflict processing: N200 responses to emotional words in a flanker task. Neuropsychologia, 48(12), 3661-3664.

Kanske, P., \& Kotz, S. A. (2011). Positive emotion speeds up conflict processing: ERP responses in an auditory Simon task. Biological psychology, 87(1), 122-127.

Kanske, P., \& Kotz, S. A. (2012). Effortful control, depression, and anxiety correlate with the influence of emotion on executive attentional control. Biological psychology, 91(1), 88-95.

Kompatsiari K., Ciardo F., Tommaso de D., \& Wykowska A. Measuring engagement elicited by eye contact in Human-Robot Interaction, 2019. In IEEE/RSJ International Conference on Intelligent Robots and Systems (IROS), Macau, China, 2019, pp. 6979-6985, doi: 10.1109/IROS40897.2019.8967747.

Kompatsiari, K., Bossi, F., \& Wykowska, A. (2021). Eye contact during joint attention with a humanoid robot modulates oscillatory brain activity. Social cognitive and affective neuroscience, 16(4), 383-392.

Kompatsiari, K., Ciardo, F., \& Wykowska, A. (2021). To follow or not to follow your gaze: The interplay between strategic control and the eye contact effect on gaze-induced attention orienting. Journal of Experimental Psychology: General. Advance online publication. https://doi.org/10.1037/xge0001074.

Kompatsiari, K., Ciardo, F., Tikhanoff, V., Metta, G., \& Wykowska, A. (2018). On the role of eye contact in gaze cueing. Scientific reports, 8(1), 1-10.

Kompatsiari, K., Pérez-Osorio, J., De Tommaso, D., Metta, G., \& Wykowska, A. (2018, October). Neuroscientifically-grounded research for improved human-robot interaction. In 2018 IEEE/RSJ International Conference on Intelligent Robots and Systems (IROS) (pp. 3403-3408). IEEE.

Larson, M. J., Clawson, A., Clayson, P. E., \& South, M. (2012). Cognitive control and conflict adaptation similarities in children and adults. Developmental Neuropsychology, 37(4), 343-357.

Logan, G. D. (1985). Executive control of thought and action. Acta Psychologica, 60, 193-210. 
Robot's social gaze affects conflict resolution

642

643

644

645

646

647

648

Mansouri, F. A., Tanaka, K., \& Buckley, M. J. (2009). Conflictinduced behavioural adjustment: A clue to the executive functions of the prefrontal cortex. Nature Reviews Neuroscience, 10, 141-152. doi:10.1038/nrn2538

Marini, F., Chelazzi, L., \& Maravita, A. (2013). The costly filtering of potential distraction: Evidence for a supramodal mechanism. Journal of Experimental Psychology: General, 142, 906-922. doi: $10.1037 / a 0029905$

Metta, G., Fitzpatrick, P., \& Natale, L. (2006). YARP: yet another robot platform. International Journal of Advanced Robotic Systems, 3(1), 8.

Metta, G., Natale, L., Nori, F., Sandini, G., Vernon, D., Fadiga, L., ... \& Montesano, L. (2010). The iCub humanoid robot: An open-systems platform for research in cognitive development. Neural networks, 23(8-9), 1125-1134.

Mullane, J. C., Corkum, P. V., Klein, R. M., \& McLaughlin, E. (2009). Interference control in children with and without ADHD: a systematic review of Flanker and Simon task performance. Child neuropsychology, 15(4), 321-342.

Padmala, S., Bauer, A., \& Pessoa, L. (2011). Negative emotion impairs conflict-driven executive control. Frontiers in Psychology, 2, 192.

Pönkänen, L. M., Peltola, M. J., \& Hietanen, J. K. (2011). The observer observed: Frontal EEG asymmetry and autonomic responses differentiate between another person's direct and averted gaze when the face is seen live. International Journal of Psychophysiology, 82(2), 180-187.

Proctor, R. W., \& Vu, K. P. L. (2006). Stimulus-response compatibility principles: Data, theory, and application. Boca Raton, FL: CRC Press

Ridderinkhof, R. K. (2002). Micro-and macro-adjustments of task set: Activation and suppression in conflict tasks. Psychological Research, 66, 312-323.

Roncone, A., Pattacini, U., Metta, G., \& Natale, L. (2016, June). A Cartesian 6-DoF Gaze Controller for Humanoid Robots. In Robotics: science and systems (Vol. 2016).

Rubichi S., Pellicano A. (2004). Does the Simon effect affect movement execution? European Journal of Cognitive Psychology, 16, 825-840.

Schellen, E., Bossi, F., \& Wykowska, A. (2021). Robot Gaze Behaviour Affects Honesty in Human-Robot Interaction. Frontiers in Artificial Intelligence, 4, 51.

Scherbaum S., Fischer R., Dshemuchadse M., Goschke T. (2011). The dynamics of cognitive control: Evidence for within-trial conflict adaptation from frequency-tagged EEG. Psychophysiology, 48, 591-600.

Schuch, S., Zweerings, J., Hirsch, P., \& Koch, I. (2017). Conflict adaptation in positive and negative mood: Applying a success-failure manipulation. Acta Psychologica, 176, 11-22. 
Robot's social gaze affects conflict resolution

675

Sellaro, R., \& Colzato, L. S. (2017). High body mass index is associated with impaired cognitive control. Appetite, 113, 301-309.

Simon, J. R., \& Rudell, A. P. (1967). Auditory SR compatibility: The effect of an irrelevant cue on information processing. Journal of Applied Psychology, 51, 300-304. doi:10.1037/ h0020586

Spatola, N., Belletier, C., Normand, A., Chausse, P., Monceau, S., Augustinova, M., ... \& Ferrand, L. (2018). Not as bad as it seems: When the presence of a threatening humanoid robot improves human performance. Science Robotics, 3(21), eaat5843.

Stroop, J. R. (1992). Studies of interference in serial verbal reactions (Reprinted from Journal Experimental-Psychology, 18, 643-662, 1935. Journal of Experimental Psychology-General, 121, 15-23. doi: 10.1037/0096-3445.121.1.15

Sturmer, B., Nigbur, R., Schacht, A., \& Sommer, W. (2011). Reward and punishment effects on error processing and conflict control. Frontiers in Psychology, 2, 335. doi: 10.3389/fpsyg.2011.00335

Van der Lubbe, R. H., \& Verleger, R. (2002). Aging and the Simon task. Psychophysiology, 39(1), 100-110.

van Steenbergen, H. (2015). Affective modulation of cognitive control: A biobehavioural perspective. In Handbook of biobehavioural approaches to self-regulation (pp. 89-107). Springer, New York, NY.

van Steenbergen, H., Band, G. P. H., \& Hommel, B. (2010). In the mood for adaptation: How affect regulates conflict-driven control. Psychological Science, 21,1629-1634. doi: $/ 10.1177 / 0956797610385951$

Vasco, V., Willemse, C., Chevalier, P., De Tommaso, D., Gower, V., Gramatica, F., Tikhanoff, V., Pattacini, U., Metta, G., Wykowska, A. (2019). Train With Me: A Study Comparing a Socially Assistive Robot and a Virtual Agent for a Rehabilitation Task. 11th International Conference on Social Robotics. Madrid, Spain, Lecture Notes in Computer Science, 11876, 10.1007/978-3-030-35888-4_42

Vygotskij, L. S. (1932). Das Problem der Altersstufen. ders.: Ausgewählte Schriften Bd, 2, pp. 53-90.

Wiese, E., Metta, G., \& Wykowska, A. (2017). Robots as intentional agents: using neuroscientific methods to make robots appear more social. Frontiers in psychology, 8, 1663.

Wykowska, A. (2020). Social Robots to Test Flexibility of Human Social Cognition. International Journal of Social Robotics, 1-9.

Xiong, A., \& Proctor, R. W. (2016). Decreasing auditory Simon effects across reaction time distributions. Journal of Experimental Psychology: Human Perception and Performance, 42(1), 23-38.

Yamaguchi, M., \& Nishimura, A. (2019). Modulating proactive cognitive control by reward: differential anticipatory effects of performance-contingent and non-contingent rewards. Psychological research, 83(2), 258-274. 
Robot's social gaze affects conflict resolution

707 Yamaguchi, M., Moore, J. D., Hendry, S., \& Wolohan, F. (2020). Exploring an Emotional Basis of Cognitive Control in the Flanker Task. https://doi.org/10.31234/osf.io/nkjy6

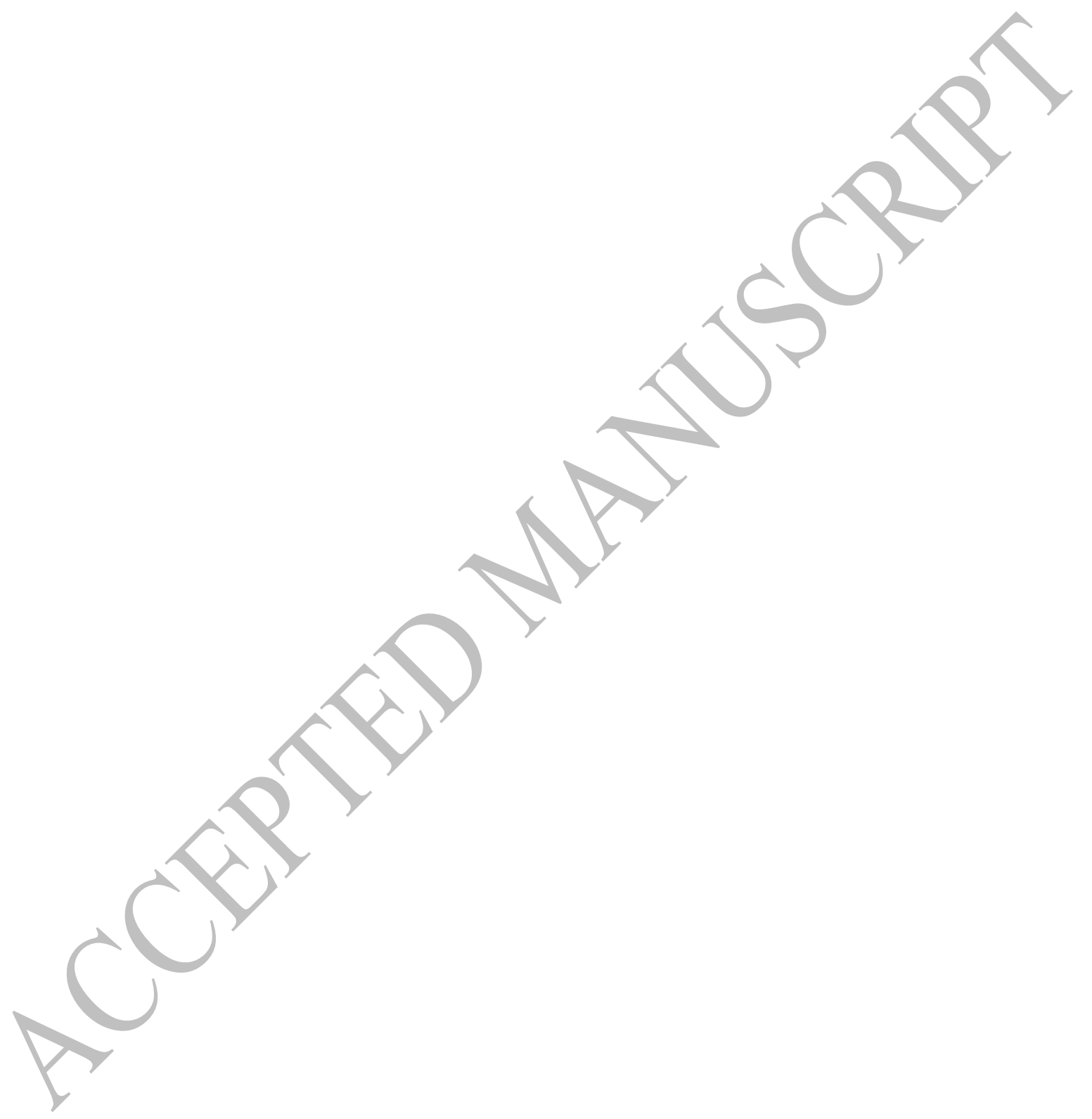

